Onkologe 2019 $\cdot 25: 392-394$

https://doi.org/10.1007/s00761-019-0560-y

(c) Springer Medizin Verlag GmbH, ein Teil von Springer Nature 2019

\author{
H.-G. Schnürch $\cdot$ M. Hampl ${ }^{2} \cdot$ H. Schmidberger ${ }^{3}$ \\ 'Kaarst, Deutschland \\ ${ }^{2}$ Klinik für Frauenheilkunde und Geburtshilfe, Universitätsklinikum Düsseldorf, Düsseldorf, Deutschland \\ ${ }^{3}$ Unimedizin Mainz, Mainz, Deutschland
}

\title{
Karzinome der Vulva und Vagina - ein Update
}

Die Zusammenfassung dieser beiden Tumorentitäten in einem Themenheft ist sinnvoll - nicht nur, weil aus Laiensicht zumeist beide als Erkrankung der "Scheide" wahrgenommen werden, sondern auch, weil sie sich in Pathogenese, Morphologie, Invasionsverhalten und klinischen Eigenschaften sehr ähneln. Nur die gering unterschiedliche anatomische Lokalisation bedingt unterschiedliche Therapiestrategien.

Beide Entitäten stellen solide Malignome mit langzeitig lokaler Infiltration und regional lymphatischer Ausbreitung dar - das erklärt die Dominanz der lokoregionären Therapiemodalitäten Operation und Bestrahlung.

\section{》) Mit vermehrtem Auftreten} der Vulvakarzinome wurde die Erarbeitung von Therapieleitlinien intensiviert

Mit dem zunehmenden Auftreten der Vulvakarzinome (von 2000 bis 2010 auf $250 \%$ ) wurde die Erarbeitung von Behandlungsleitlinien intensiviert und mündete im Jahr 2015 in den aktualisierten S2k-Leitlinien der AGO (Arbeitsgemeinschaft Gynäkologische Onkologie) aus DGGG (Deutsche Gesellschaft für Gynäkologie und Geburtshilfe) und DKG (Deutsche Krebsgesellschaft). Auch wenn für die Vaginalkarzinome die Häufigkeitszunahme so nicht zu verzeichnen war (relativ konstant etwa 500 Fälle pro Jahr in Deutschland), so wurde dennoch die Abdeckung mit einer aktuellen S2kLeitlinie in 2018 erreicht.
Mit diesen Leitlinien besteht ein Gerüst an konsentierten Empfehlungen für die Diagnostik und Behandlung wie bei den häufigeren Tumoren - die gynäkologischen Tumorentitäten sind damit sämtlich in AWMF-Leitlinien abgebildet. Dadurch erhält die Behandlung der gynäkologischen Tumorpatienten eine solide Grundlage durch zusammengetragene wissenschaftliche Evidenzen und Expertenwissen, die für die klinische Tätigkeit der Tumorbehandlungszentren von großer Bedeutung ist. Die Organisation der Krebsbehandlung in Deutschland durch die DKG und die medizinischen Fachgesellschaften hat in den vergangenen 15 Jahren über eine strenge Zertifizierung hin zu spezialisierten klinischen Zentren mit höherer Fallzahl geführt. Dadurch können wir uns dem Ziel einer hochqualitativen Tumorbehandlung in Deutschland weiter nähern.

Die Artikel in diesem Heft beschäftigen sich schwerpunktmäßig mit den Aspekten von Diagnostik und Therapie der beiden Tumorentitäten Vulva- und Vaginalkarzinom, die einerseits die aktuellen Anforderungen schildern und andererseits Neues anbieten, wie z.B. die Sentineltechnik beim Vaginalkarzinom.

Alt-Radtke wird zur Diagnostik in Form von Sonographie der Lymphknotenregionen, MRT, CT und PET-CT bei Vulva bzw. Vaginalkarzinom den aktuellen Stand darstellen.

Im Beitrag von Hampl und Dannecker wird die Möglichkeit der Primärprävention des Vulva- und Vaginalkarzinoms und seiner Vorstufen durch die HPVImpfung dargestellt. Da ein Teil dieser Tumoren HPV-induzierte Läsionen darstellen, kann in diesen Fällen eine pri- 
Hier steht eine Anzeige.

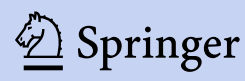


märe Verhinderung der Entstehung dieser Läsionen mit der prophylaktischen Impfung erfolgen.

Die Wächterlymphknotentechnik hat inzwischen festen Einzug in die Therapie des Vulvakarzinoms gehalten. Aber wie sieht es beim Vaginalkarzinom aus? Ist diese Technik auch dort einsetzbar? Dazu werden die Autoren Wölber und Schnürch unter Einbezug der gerade veröffentlichtenLeitliniezur „Diagnostikund Therapie des Vaginalkarzinoms"Stellung nehmen.

Bei fortgeschrittenen Tumoren der Vulva und v. a. der Vagina ist die Strahlentherapie ein fester Bestandteil der Therapie. Marnitz und Lindell zeigen die Indikationen zur Strahlentherapie auf.

Die Studienlandschaft bei Vulva- und Vaginalkarzinomen ist spärlich, dazu wird Barinoff Stellung nehmen und die wenigen Studien auflisten.

Die Systemtherapie dieser Tumoren ist im metastasierten Stadium indiziert, aber leider gibt es auch hier nur wenige randomisierte Studien und nur mäßige Erfolge. Ob sich das mit den neuen Antikörper(AK)-Therapien ändert, wird Hagen versuchen zu beantworten.
Bedeutsam für zukünftige Erkenntnisse ist in diesem Zusammenhang auch die beschriebene Einrichtung von Registerstellen für seltenere Tumoren, die uns eines Tages in die Lage versetzen können, das reine Expertenwissen durch bisher nicht erreichbare Evidenz zu unterfüttern.

Für die Schriftleiter Monika Hampl

Für die Herausgeber Heinz Schmidberger

\section{Korrespondenzadresse}

Prof. Dr. M. Hampl
Klinik für Frauenheilkunde
und Geburtshilfe,
Universitätsklinikum
Düsseldorf
Moorenstr. 5, 40225 Düssel-
dorf, Deutschland
hampl@med.uni-
duesseldorf.de

Interessenkonflikt. H.-G. Schnürch, M. Hampl und $\mathrm{H}$. Schmidberger geben an, dass kein Interessenkonflikt in Bezug auf dieses Thema besteht.

\section{Der neue Kurs zu NSCLC}

Alles Wichtige kompakt und aktuell auf den Punkt gebracht! 